\title{
Exploring the Role of Macroeconomic Mechanisms in Voluntary Resource Provisioning in Community Network Clouds
}

\author{
Amin M. Khan and Felix Freitag \\ Department of Computer Architecture \\ Universitat Politècnica de Catalunya \\ Barcelona, Spain \\ $\{$ mkhan, felix\}@ac.upc.edu
}

\begin{abstract}
Internet and communication technologies have lowered the costs of enabling individuals and communities to collaborate together. This collaboration has provided new services like user-generated content and social computing, as evident from success stories like Wikipedia. Through collaboration, collectively built infrastructures like community wireless mesh networks where users provide the communication network, have also emerged. Community networks have demonstrated successful bandwidth sharing, but have not been able to extend their collective effort to other computing resources like storage and processing. The success of cloud computing has been enabled by economies of scale and the need for elastic, flexible and on-demand provisioning of computing services. The consolidation of today's cloud technologies offers now the possibility of collectively built community clouds, building upon usergenerated content and user-provided networks towards an ecosystem of cloud services. We explore in this paper how macroeconomic mechanisms can play a role in overcoming the barriers of voluntary resource provisioning in such community clouds, by analysing the costs involved in building these services and how they give value to the participants. We indicate macroeconomic policies and how they can be implemented in community networks, to ease the uptake and ensure the sustainability of community clouds.
\end{abstract}

Keywords: community cloud, cloud computing, community networks, economic mechanisms, collaborative resource sharing

\section{Introduction}

Recent developments in communication technologies like Internet, email and social networking have significantly removed the barriers for communication and coordination for small to large groups bringing down the costs that obstructed collaborative production before the era of Internet [1]. The ICT revolution ushered in group communication and collaborative production with popular applications now widely adopted, like social networking, social bookmarking, usergenerated content, photo sharing, and many more. Even infrastructures based 
on a cooperative model have been built, for example community wireless mesh networks gained momentum in early 2000s in response to limited options for network connectivity in rural and urban communities [2]. Using off-the-shelf network equipment and open unlicensed wireless spectrum, volunteers set up wireless networks in their local communities to provide network and communication infrastructure. These wireless networks have proved quite successful, for example Guifinet ${ }^{1}$ provides wireless and optical fibre based broadband access to more than 20,000 users. Community networks successfully operate as IP networks, since the nodes' bandwidth is shared among all the members in a reciprocal manner.

Despite achieving sharing of bandwidth, community networks have not been able to extend this sharing to other computing resources like storage. There are not many applications and services used by members of community networks that take advantage of resources available within community networks. Community networks are based on voluntary contributions of participants, and economic or social incentives to encourage this have been crucial to achieve the sustainability of the community networks [3]. Apparently the current incentives in community networks are not sufficient enough to overcome the barriers for realising the sharing of other computing resources besides just bandwidth.

Sharing of computing resources in the Internet is now commonplace because of the wide adoption of cloud computing model [4]. Cloud computing provides on-demand, elastic, flexible and cost-effective access to computing resources. Today's clouds are mainly provided upon a pay-per-use model, where the cloud services are offered to the consumers as a utility and by commercial providers. Cloud computing allows enterprises and individuals to reduce significantly the time and capital investment in setting up their own infrastructure. Instead, they can request resources on demand from the cloud services providers, which not only lowers the total cost of ownership for consuming resources because of economies of scale, but leaving low level details to the service providers focus can be shifted towards building and using high level applications. This also applies that an individual or organisation is no longer limited by the resources present locally and owned directly. When demand exceeds the current capacity, more resources can be requested on the fly from one or more cloud services providers. This has relevance for community networks as the members in aggregate boast much more resources than owned by a single individual or a small group. When members of community network can share and trade resources based on a cloud computing model, they can sell their excess capacity as the demand fluctuates and in return can take advantage of services and applications that were not possible earlier due to the limited resources locally.

The concept of community clouds has been introduced in its generic form before, e.g. $[5,6]$, as a cloud deployment model in which a cloud infrastructure is built and provisioned for an exclusive use by a specific community of consumers with shared concerns and interests. We refer here to a specific kind of a community cloud in which sharing of computing resources is from within com-

\footnotetext{
${ }^{1}$ http://guifi.net
} 
munity networks, using the application models of cloud computing in general. Members of community network can share and trade resources, they can sell their excess capacity as the demand fluctuates and in return can take advantage of services and applications that the community cloud enables, which were not possible earlier due to the limited resources on the users' local machines. Realising community cloud involves a lot of challenges both in technological and socio-economic context, but also promises interesting value proposition for communities in terms of local services and applications.

Our main objective in this paper is to explore the macroeconomic mechanisms that can help in adoption and growth of community cloud model. We contribute first a cost-value proposition describing the conditions under which community clouds should emerge. Secondly, we propose a set of macroeconomic policies that, if placed in community networks, should accelerate the uptake and help the sustainability of community clouds. We elaborate on this in the rest of the paper as follows. Section 2 introduces possible cloud scenarios in community networks. Section 3 discusses our cost-value proposition of community clouds, and section 4 proposes different macroeconomic mechanisms for community clouds. Section 5 concludes and indicates future work.

\section{Cloud Scenarios in Community Networks}

We consider clouds in community networks, a community cloud that provides services built from using resources available from within the community networks and owned and managed by the members of the community networks themselves. Such a community cloud infrastructure that is deployed in real community networks needs to be designed according to the conditions and characteristics of community networks, which also determine the most likely scenarios for these community clouds.

\subsection{Background on Community Networks}

A community network like Guifi.net is organised into zones where a zone can be a village, a small city, a region, or districts of a larger city. Mostly, the detailed technical support for the members is only available within the community of their zone [7], so we identify a zone to have the highest social strength within the community network. The computer machines or nodes in a community network vary widely in their capacity, function and capability, as illustrated in Figure 1. Some hardware is used as super nodes that have multiple wireless links and connect with other super nodes to form the backbone of the community network [7]. Others act just as clients and are only connected to the access point of a super node. As depicted in Figure 1, resources for the community cloud can be attached to the networking nodes. 


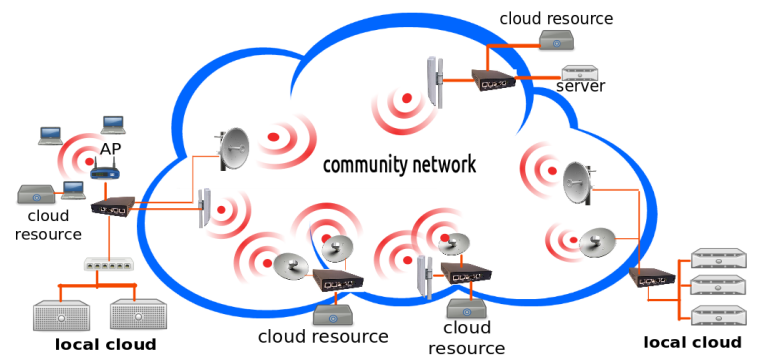

Fig. 1. Nodes in a community network with cloud resources

\subsection{Local Community Cloud}

The cohesive nature of zones gives rise to the scenario of the local community cloud, interpreting the characteristics of the social networks existing within zones and the topology of the community network. In this scenario, some super nodes with their better connectivity and high availability are responsible for the management of a set of attached nodes that are contributing cloud resources.

\subsection{Federated Community Cloud}

Local community cloud can provide services for the users within its zone. Multiple cloud nodes from different zones in a community network, however, can participate together in a federated community cloud to support greater functionality and higher capacity. The nodes in a given zone are directly managed by a super node in that zone but they can also consume resources from other zones, given that there is a coordination mechanism among zones in place. Within an economic context, the local community cloud is an example of a virtual organisation, and the federated scenario represents the peering agreements between multiple virtual organisations.

\section{Cost and Value Relationships in Community Cloud}

The community clouds can be seen as private enterprises with private provisioning of public goods. This model can suffer from social dilemmas, like the tragedy of the commons, meaning that free riding and under-provisioning will destroy the system in the absence of any mechanisms to overcome these issues. The socio-economic context of community networks implies that mechanisms that foresee social exclusion can be effective to direct the users' behaviour [8].

Figure 2 shows the desired relationship between the cost and value proposition as the community cloud evolves and gets adopted by wider audience. In 

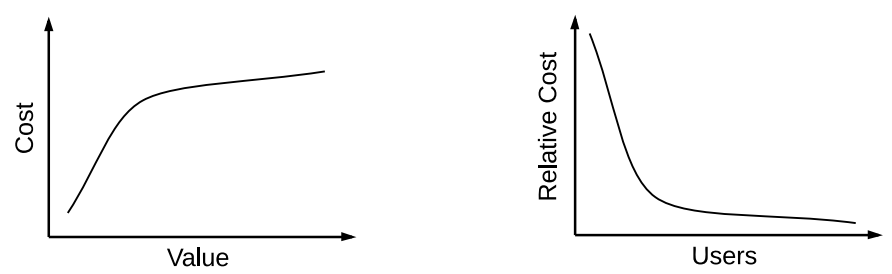

Fig. 2. Relationship between cost and value in evolution of community cloud

the nascent stage, the community cloud will not be able to provide much value until a critical mass of users are using the system. After that threshold, still the relative cost to achieve a little utility will be significant, which means that the early adopters of the system remain highly motivated and committed to the success of community cloud and continue to contribute resources even though they receive little value from the system in return. But once a significant proportion of the overall population has joined the community cloud, the relative cost to obtain value from the system tumbles and in the longer run the system is able to sustain itself with contributions that may be small in size but are made by a large number of users. The objective of the economic mechanisms and the social and psychological incentives is to let the system transition from inception through early adoption to finally ubiquitous usage.

\subsection{Costs for Participation}

The initial costs for setting up nodes in the community cloud involves hardware costs including the price of the computing and networking equipment, and installation costs including the manual labour needed. The continuous operation of the cloud node requires additional costs including network costs given by donating network bandwidth and any other subscription fees, energy costs to pay for electricity bills to run the computer equipment as well as cooling apparatus, maintenance cost to fund any technical support and replacements for parts, and hosting costs to provide storage space for the equipment. Besides these costs at the individual level, there are also the transaction costs [9] or management overheads to direct the group coordination and collaborative production efforts necessary for the operation of community cloud.

\subsection{Value Proposition}

The individuals in community cloud act as private enterprises where they offer services to generate revenue. The revenue for the community cloud users include tangible benefits like the services and applications that they will be able to consume, and intangible benefits like the sense of belonging to the community and personal satisfaction because of their contributions. The services can range from infrastructure to platform to software services meeting a spectrum of different needs of the users. Once community cloud gets adopted by a critical 
mass, community may also generate revenue by offering computing resources to commercial enterprises, similar to selling excess power capacity in the case of Smart Grid. For example, community can get into partnership agreements with the ICT providers where community can buy network bandwidth in return for providing access to the computing resources of the community cloud.

\subsection{Comparison with Commercial Services}

We discuss the community cloud cost and value in comparison with two popular commercial services that are also based in part on the idea of reciprocal sharing, Spotify $^{2}$ and Skype ${ }^{3}$. Spotify is a subscription-based music streaming service which reduces its infrastructure and bandwidth costs by serving cached content from users' devices as well as its own servers. Skype is a communication service which uses caches on users' devices for storing and processing information required for managing the underlying infrastructure. Both Spotify and Skype offer free as well as paid services. Why do users agree to contribute resources, and even when they are paying for the service?

An argument is that the costs for users are minimal. Both services mostly consume storage, computation time, power and bandwidth on the users' devices. Since these resources are not very expensive and the services' usage remains relatively low, the users do not mind this arrangement or not even notice it. But even more important, these services are designed so intuitively that most users do not even realise about donating the resources, and even when they do, the value these services provide has sufficient incentive.

The success of such services implies that for community cloud as well, the users should be able to join with zero or very little costs. The value proposition of the community cloud services should be strong enough to attract early adopters and keep them committed. The economic mechanisms in place for encouraging reciprocal sharing and ensuring overall system health and stability should be either invisible for non-technical users or very simple to understand and work with.

\section{Design of Macroeconomic Policies}

We discuss in this section the macroeconomic policies we propose for community clouds, addressing relevant issues of the technical, social, economic and legal aspects of the community cloud system. We approach the problem by having explored some of the mechanisms previously in simulations [10] and also by developing a prototype implementation which is currently deployed in the Guifi community network [11] and which will allow to get users involved and participating in a real world scenario.

\footnotetext{
2 http://www.spotify.com

${ }^{3}$ http://www. skype.com
} 


\subsection{Commons License}

The agreement and license to join a community cloud should encourage and help enforce reciprocal sharing for community clouds to work. The Wireless Commons License $^{4}$ or Pico Peering Agreement ${ }^{5}$ is adopted by many community networks to regulate network sharing. This agreement could serve as a good base for drafting an extension that lays out the rules for community clouds.

\subsection{Peering Agreements}

When different community clouds federate together, agreements should ensure fairness for all the parties. Agreements between different communities should describe the rules for peering between clouds. Within such agreements, local currency exchanges could be extended to address cases of imbalance in contribution across different zones [12].

\subsection{Ease of Use}

The easier it is for users to join, participate and manage their resources in the community cloud, the more the community cloud model will be adopted. This requires lowering the startup costs and entry barriers for participation. To this end, in terms of an institutional policy, we have developed a Linux-based distribution ${ }^{6}$, to be used in the Guifi.net community cloud [11]. It will make the process of joining and consuming cloud services almost automated with little user intervention. This effect will make the community cloud appealing to nontechnical users.

\subsection{Social Capital}

Community clouds need to appeal to the social instincts of the community instead of solely providing economic rewards. This requires maximising both bonding social capital [13] within local community clouds in order to increase the amount of resources and commitment of the users, and bridging social capital in order to ensure strong cooperation between partners in federated community clouds. Research on social cloud computing [14] has already shown how to take advantage of the trust relationships between members of social networks to motivate contribution towards a cloud storage service.

\subsection{Transaction Costs}

The community cloud, especially in its initial stages, will require strong coordination and collaboration between early adopters as well as developers of cloud

\footnotetext{
${ }^{4}$ http://guifi.net/es/ProcomunXOLN

${ }^{5}$ http://www.picopeer.net

${ }^{6}$ http://repo.clommunity-project.eu
} 
applications and services, so we need to lower the transaction costs for group coordination [9]. This can take advantage of existing Guifinet's mailing list ${ }^{7}$, but also of the regular social meetings and other social and software collaboration tools. It also requires finding the right balance between a strong central authority and decentralised and autonomous mode of participating for community members and software developers.

\subsection{Locality}

Since the performance and quality of cloud application in community networks can depend a lot on the locality, applications need to be network and location aware, but this also requires that providers of resources should honour their commitment to local community cloud implying that most requests are fulfilled within the local zone instead of being forwarded to other zones. We have explored the implications of this earlier when studying the relationship between federating community clouds $[10,15]$.

\subsection{Overlay Topology}

Community networks are an example of scale-free small-world networks [7], and the community cloud that results from joining community networks users is expected to follow the same topology and inherit characteristics similar to scalefree networks. As the overlay between nodes in the community cloud gets created dynamically [16], the community cloud may evolve along different directions as users of the underlying community network join the system. As the applications in community cloud will most likely be location and network aware to make the most efficient use of the limited and variable resources in the network, the overlay steered concentration and distribution of consumers and providers of services direct the state and health of the community cloud.

\subsection{Entry Barriers}

In order to control the growth of the community cloud and provide a reasonable quality of experience for early adopters and permanent users, different approaches can be considered, for example, a community cloud open to everyone, by invitation only, or one that requires a minimum prior contribution.

\subsection{Role of Developers}

The developers of the cloud applications are expected to play an important intermediary role between providers of resources and consumers of services, for example adding value to the raw resources and selling them to consumers at a premium. End users could have both the roles of raw resource providers and consumers which find the value of the cloud in the provided applications.

\footnotetext{
${ }^{7}$ http://guifi.net/en/forum
} 


\subsection{Service Models}

Cloud computing offers different service levels, infrastructure, platform and softwareas-a-service (SaaS). Similar to the three economic sectors for provisioning goods, the third level, the SaaS of the cloud reaches the end users. For providing value from the beginning in the community cloud, we propose to prioritize provisioning SaaS at the early stage of the community cloud.

\subsection{Value Addition and Differentiation}

The community cloud requires services that provide value for users. In addition, these services need to compete and differentiate from the generic cloud services available over the Internet. In this line, FreedomBox ${ }^{8}$ services focus on ensuring privacy, and FI-WARE CoudEdge ${ }^{9}$ and ownCloud ${ }^{10}$ let cloud applications consume resources locally.

\section{Conclusion and Future Work}

Community clouds take advantage of resources available within community networks for realising cloud-based services and applications tailored to local communities. Being community clouds a case of private provisioning of public goods, economic mechanisms and policies are needed to direct their growth and sustainability. First, we identified the cost and value evolution of the community cloud during its emergence and under permanent operation. A core number of highly motivated contributors is needed at the beginning. Once the community cloud is operational, its value should easily exceed the cost of the minor contribution expected from the users. The socio-economic context of community networks forms the basis for the macroeconomic policies that we proposed for community clouds. We outlined and illustrated these policies that address technical, social, economic and legal aspects of the community cloud system.

Based on the proposed macroeconomic policies, our next step is to design and integrate them in our prototype implementation of the community cloud that we currently deploy in the real-world Guifi.net community network. The resulting empirical studies will help assessing the effect of the proposed economic mechanisms further. Our hope is that community clouds will complement the existing public cloud services paving the way for innovative and interesting applications for local communities.

\section{Acknowledgement}

This work was supported by the European Framework Programme 7 FIRE Initiative projects CONFINE, FP7-288535, and CLOMMUNITY, FP7-317879, and

\footnotetext{
${ }^{8}$ http://freedomboxfoundation.org

${ }^{9}$ http://catalogue.fi-ware.eu/enablers/cloud-edge

10 http://owncloud.org
} 
by the Universitat Politècnica de Catalunya BarcelonaTech and the Spanish Government through the Delfin project, TIN2010-20140-C03-01.

\section{References}

1. Shirky, C.: Here Comes Everybody: The Power of Organizing Without Organizations. Penguin Group (2008)

2. Braem, B., et al.: A case for research with and on community networks. ACM SIGCOMM Computer Communication Review 43(3) (July 2013) 68-73

3. Bina, M., Giaglis, G.: Unwired Collective Action: Motivations of Wireless Community Participants. In: International Conference on Mobile Business (ICMB'06), Copenhagen, Denmark, IEEE (June 2006) 31-40

4. Buyya, R., Broberg, J., Goscinski, A.: Cloud Computing: Principles and Paradigms. John Wiley \& Sons, Inc. (2011)

5. Mell, P., Grance, T.: The NIST Definition of Cloud Computing. NIST Special Publication 800(145) (2011)

6. Marinos, A., Briscoe, G.: Community Cloud Computing. Cloud Computing 5931 (2009) 472-484

7. Vega, D., Cerda-Alabern, L., Navarro, L., Meseguer, R.: Topology patterns of a community network: Guifi.net. In: 1st International Workshop on Community Networks and Bottom-up-Broadband (CNBuB 2012), within IEEE WiMob, Barcelona, Spain (October 2012) 612-619

8. Greiff, M.: Rewards and the private provision of public goods on dynamic networks. Journal of Evolutionary Economics 23(5) (September 2013) 1001-1021

9. Coase, R.H.: The Nature of the Firm. Economica 4(16) (November 1937) 386-405

10. Khan, A.M., Büyükşahin, U.C., Freitag, F.: Towards Incentive-Based Resource Assignment and Regulation in Clouds for Community Networks. In Altmann, J., Vanmechelen, K., Rana, O.F., eds.: GECON 2013. Volume 8193 of LNCS. Springer (September 2013) 197-211

11. Jiménez, J., et al.: Deploying PaaS for Accelerating Cloud Uptake in the Guifi.net Community Network. In: IEEE International Workshop on the Future of PaaS 2014, Boston, Massachusetts, USA (March 2014)

12. Punceva, M., Rodero, I., Parashar, M., Rana, O.F., Petri, I.: Incentivising resource sharing in social clouds. Concurrency and Computation: Practice and Experience (March 2013)

13. Coleman, J.S.: Social capital in the creation of human capital. American Journal of Sociology 94 (1988) pp. S95-S120

14. Chard, K., Bubendorfer, K., Caton, S., Rana, O.F.: Social Cloud Computing: A Vision for Socially Motivated Resource Sharing. IEEE Transactions on Services Computing 5(4) (January 2012) 551-563

15. Khan, A.M., Büyükşahin, U.C., Freitag, F.: Prototyping Incentive-based Resource Assignment for Clouds in Community Networks. In: 28th International Conference on Advanced Information Networking and Applications (AINA 2014), Victoria, Canada, IEEE (May 2014)

16. Nakao, A., Wang, Y.: On Cooperative and Efficient Overlay Network Evolution Based on a Group Selection Pattern. IEEE Transactions on Systems, Man, and Cybernetics, Part B: Cybernetics 40(2) (April 2010) 493-504 